\begin{tabular}{c} 
International Journal of Engineering \& Technology, $7(3)(2018) 1243-1244$ \\
International Journal of Engineering \& Technology \\
SPC \\
Website: $\begin{array}{c}\text { www.sciencepubco.com/index.php/IJET } \\
\text { doi: } 10.14419 / \text { ijet. } v 73.13269 \\
\text { Research paper }\end{array}$ \\
\hline
\end{tabular}

\title{
Gutman Index and Harary Index of Unitary Cayley Graphs
}

\author{
Roshan Sara Philipose $^{1 *}$ and Sarasija P.B ${ }^{2}$ \\ ${ }^{1}$ Research Scholar, Department of Mathematics, Noorul Islam Centre For Higher Education, Kumaracoil-629175 \\ ${ }^{2}$ Professor, Department of Mathematics, Noorul Islam Centre For Higher Education, Kumaracoil-629175 \\ ${ }^{*}$ Corresponding author E-mail:roshanjilu@gmail.com
}

\begin{abstract}
In this paper, we determine the Gutman Index and Harary Index of Unitary Cayley Graphs. The Unitary Cayley Graph $X_{n}$ is the graph with vertex set $V\left(X_{n}\right)=\left\{u \mid u \in Z_{n}\right\}$ and edge set $\left\{u v \mid \operatorname{gcd}(u-v, n)=1\right.$ and $\left.u, v \in Z_{n}\right\}$, where $Z_{n}=\{0,1, \ldots, n-1\}$

Keywords: Complete Graph; Gutman Index; Harary Index; Topological index; Unitary Cayley Graphs.
\end{abstract}

\section{Introduction}

The general concepts of graph theory can be viewed in [2]. Here, we consider the Unitary Cayley Graph $X_{n}=\operatorname{Cay}\left(Z_{n} ; U_{n}\right)$ where $Z_{n}$ is the additive group of intergers modulo $n$ and $U_{n}$ is the multiplicative group of its units $(n>1)$. Therefore, its vertex set comprises of elements $u$ in $\{0,1, \ldots, n-1\}$ and $u, v$ are adjacent if and only if $\operatorname{gcd}(u-v, n)=1 . X_{n}$ is $\phi(n)$-regular where $\phi(n)=\left|U_{n}\right|$. Also, it is complete when $n$ is prime $p$ and complete bipartite when $n$ is a prime power $p^{t}$ (for the properties of unitary Cayley graphs, see [4]).

A topological index, also known as graph-theoretic index, is graph invariant and is a type of molecular descriptor [3]. Several distancebased and degree-based topological indices have been defined. Among them, we choose 2 distance based topological indicesGutman Index and Harary Index for the computation of the respective indices of Unitary Cayley graphs.

Gutman proposed the idea of Gutman Index Gut $(\mathrm{G})$ (Schultz index of the $2^{\text {nd }}$ kind) of a connected undirected graph $\mathrm{G}$ in 1994 [1] and it is defined as

$\operatorname{Gut}(G)=\sum_{u, v \in V(G)} d(u) d(v) d_{G}(u, v)$.

Plavšić et.al introduced the Harary index [5] of a graph $G$ on $n$ vertices in 1993 and it is defined as

$H(G)=\sum_{u, v \in V(G)} \frac{1}{d_{G}(u, v)}$

In both defintions, the summation goes over all unordered pairs of vertices of $G, V(G)$ represents the vertex set of graph $G$ and $d_{G}(u, v)$ denotes the number of edges in a shortest path connecting vertices $u$ and $v$. Also, $d(u)$ and $d(v)$ denote the degrees of vertices $u$ and $v$. In this paper, the folowing two lemmas (for the proof, see [4]) are applied for the computation.

Lemma 1.1: The Unitary Cayley graph $X_{n}, n \geq 2$, is bipartite if and only if $n$ is even.
Lemma 1.2: For integers $n \geq 2$, $a$ and $b$, denote by $F_{n}(a-b)$ the number of common neighbours of distinct vertices $a, b$ in the Unitary Cayley graph $X_{n}$ is given by

$F_{n}(a-b)=n \prod_{p / n}\left(1-\frac{\varepsilon(p)}{p}\right)$

where $\varepsilon(p)=\left\{\begin{array}{ll}1, & \text { if } p \text { divides }(a-b) \\ 2, & \text { if } p \text { doesnot divide }(a-b)\end{array}\right.$ (p is prime).

\section{Gutman Index of Unitary Cayley Graphs}

In this section, Gutman Index of the Unitary Cayley graphs is determined.

Theorem 2.1: Let $X_{n}$ be the Unitary Cayley graph on $n$ vertices. Then for an integer $n \geq 2$, we deduce:

1. if $\mathrm{n}$ is prime, $\operatorname{Gut}\left(X_{n}\right)=\frac{n(n-1)^{3}}{2}$.

2. if $n=2^{r}$ and $r>1, \operatorname{Gut}\left(X_{n}\right)=\frac{n^{3}(3 n-4)}{16}$.

3. if $\mathrm{n}$ is odd but not prime, $\operatorname{Gut}\left(X_{n}\right)=\frac{n \phi(n)^{2}[2(n-1)-\phi(n)]}{2}$.

4. if $\mathrm{n}$ is even and has an odd prime divisor, $\operatorname{Gut}\left(X_{n}\right)=$ $\frac{n \phi(n)^{2}[5 n-4(\phi(n)+1)]}{4}$.

Proof: Let $X_{n}$ be the Unitary Cayley graph and $X_{n}$ is $\phi(n)$-regular.

1. Suppose $n$ is prime $p$.

Then $X_{p}=K_{p}$, a complete graph.

Therefore, by definition of $G u t(G)$ and $H(G)$,

$$
\begin{array}{r}
\operatorname{Gut}\left(X_{n}\right)=\underbrace{\phi(n)^{2}+\phi(n)^{2}+\cdots+\phi(n)^{2}}_{\frac{n(n-1)}{2}} \\
=(n-1)^{2} \cdot\left[\frac{n(n-1)}{2}\right] \\
=\frac{n(n-1)^{3}}{2} .
\end{array}
$$


2. Suppose $n=2^{r}$ and $r>1$.

Then $X_{n}=K_{n / 2, n / 2}$, a complete bipartite graph with $V\left(X_{n}\right)=$ $V(A U B) ; A=\{0,2, \ldots, n-2\}, B=\{1,3, \ldots, n-1\}$.

Therefore, by applying lemma 1.2 , we obtain the distance between $(n / 2)^{2}$ pairs of vertices as 1 and distance between $\frac{n(n-2)}{4}$ pairs of vertices as 2 .

$$
\text { So, } \begin{aligned}
\operatorname{Gut}\left(X_{n}\right) & =\sum_{u, v \in V\left(X_{n}\right)} d(u) d(v) d_{G}(u, v) \\
& =\sum_{u, v \in V\left(X_{n}\right)} d(u) d(v)+2 \sum_{u, v \in V\left(X_{n}\right)} d(u) d(v) \\
& =(n / 2)^{2} \sum 1+(n / 2)^{2} \sum 2 \\
& =(n / 2)^{2} \cdot n^{2} / 4+2(n / 2)^{2} \cdot \frac{n(n-2)}{4} \\
& =\frac{n^{3}(3 n-4)}{16}
\end{aligned}
$$

3. Suppose $n$ is odd but not prime.

i.e., $n=\left(p_{1}\right)^{\alpha_{1}}\left(p_{2}\right)^{\alpha_{2}} \cdots\left(p_{r}\right)^{\alpha_{r}} ; p_{i} \neq 2$ and $1 \leq i \leq r$. Therefore, we can infer that to every pair of distinct vertices, there exists a common neighbour by lemma 1.2.

Then distance between $\frac{n \phi(n)}{2}$ pairs of vertices is 1 and distance between $\frac{n[n-(\phi(n)+1)]}{2}$ pairs of vertices is 2 .

$$
\begin{aligned}
\operatorname{Gut}\left(X_{n}\right) & =\sum_{u, v \in V\left(X_{n}\right)} d(u) d(v) d_{G}(u, v) \\
& =\sum \phi(n)^{2}+2 \sum \phi(n)^{2} \\
& =\phi(n)^{2} \cdot\left[\frac{n \phi(n)}{2}\right]+2 n \phi(n)^{2} \cdot\left[\frac{n-(\phi(n)+1)}{2}\right] \\
& =\frac{n \phi(n)^{2}[2(n-1)-\phi(n)]}{2} .
\end{aligned}
$$

4. Suppose $n$ is even and has an odd prime divisor $p$. Then $X_{n}$ is bipartite with vertex partition $A=\{0,2, \ldots, n-2\}$ and $B=$ $\{1,3, \ldots, n-1\}$. Also, $d(u)=d(v)=\phi(n)$ since $X_{n}$ is $\phi(n)$ regular.

Claim: Calculate $d_{G}(u, v)$

To obtain $d_{G}(u, v)$, consider 2 cases.

Case 1: Consider $u \in A$.

Taking $v \in A$, we obtain a common neighbour by lemma 1.2. Thus $\mathrm{d}_{G}(u, v)=2$. Taking $v \in B$, we obtain $d_{G}(u, v)=1$ and $d_{G}(u, v)=3$ by considering $\mathrm{B}$ as the union of 2 sets $B_{1}$ and $B_{2}$ comprising of elements adjacent to $u$ and non-adjacent to $v$ respectively.

Case 2: Consider $u \in B$.

Similarly, we obtain $d_{G}(u, v)$ as 1,2 and 3 when $v \in B_{1}, v \in A$ and $v_{2}$ respectively.

$$
\begin{aligned}
\operatorname{Gut}\left(X_{n}\right) & =\sum_{u, v \in V\left(X_{n}\right)} d(u) d(v) d_{G}(u, v) \\
& =\sum_{u, v \in V\left(X_{n}\right)} d(u) d(v)+2 \sum_{u, v \in V\left(X_{n}\right)} d(u) d(v)+
\end{aligned}
$$

$3 \sum_{u, v \in V\left(X_{n}\right)} d(u) d(v)$

$$
=\frac{\phi(n)^{2} \cdot n \phi(n)}{2}+2 \frac{\phi(n)^{2} \cdot\left(n^{2}-2 n\right)}{4}+
$$

$3 \frac{\phi(n)^{2}[n / 2-\phi(n)] n}{2}$

$$
=\frac{n \phi(n)^{2}[5 n-4(\phi(n)+1)]}{4} .
$$

\section{Harary Index of Unitary Cayley Graphs}

We determine Harary Index of Unitary Cayley graphs in this section. Theorem 3.1: For the Unitary Cayley graph $X_{n}(n>1)$, the Harary Index ,

$H\left(X_{n}\right)= \begin{cases}\frac{n(n-1)}{2}, & n \text { is prime } \\ \frac{n(3 n-2)}{8}, & n=2^{r} \text { and } r>1 \\ \frac{n(\phi(n)+n-1)}{4}, & n \text { is odd but not prime } \\ \frac{n[5 n+2(4 \phi(n)-3)]}{24}, & n \text { is even and has an odd prime divisor }\end{cases}$

Proof: For $n$ is prime, we get a complete graph $X_{n}$. So by definition, $H\left(X_{n}\right)=\underbrace{1+1 \cdots+1}_{\frac{n(n-1)}{2}}=\frac{n(n-1)}{2}$.

For $n=2^{r}$ and $r>1$, we get a biclique $X_{n}$ with vertex partition. Thus $H\left(X_{n}\right)=\frac{n(3 n-2)}{8}$.

For $n$ is odd but not prime, we get $d_{G}(u, v)$ as 1 and 2 (using lemma 1.2) respectively.

$$
\text { Thus, } \begin{aligned}
H\left(X_{n}\right) & =\sum_{u, v \in V\left(X_{n}\right)} \frac{1}{d_{G}(u, v)} \\
& =\frac{n \phi(n)}{2}+1 / 2 \cdot \frac{n[n-(\phi(n)+1)]}{2} \\
& =\frac{n[\phi(n)+n-1]}{4} .
\end{aligned}
$$

For $n$ is even and has an odd prime divisor, we get a bigraph $X_{n}$. Then it can be easily understood from theorem 2.1 that $d_{G}(u, v)$ is 1 , 2 and 3 respectively.

$$
\text { Thus, } \begin{aligned}
H\left(X_{n}\right) & =\sum_{u, v \in V\left(X_{n}\right)} \frac{1}{d_{G}(u, v)} \\
& =\sum \frac{1}{1}+\sum \frac{1}{2}+\sum \frac{1}{3} \\
& =\frac{n \phi(n)}{2}+\frac{n^{2}-2 n}{8}+\frac{n[n / 2-\phi(n)]}{6} \\
& =\frac{n[5 n+2(4 \phi(n)-3)]}{24} .
\end{aligned}
$$

\section{Conclusion}

In this paper, terminologies used were discussed as well. Moreover, the Gutman Index and Harary index of Unitary cayley graphs $X_{n}$ were deduced for an integer $n \geq 2$.

\section{Acknowledgement}

We are grateful to all who provided insight and shared their comments that greatly improved the manuscript.

\section{References}

[1] I. Gutman, "Selected Properties of the Schultz Molecular Topological Index", J. Chem. Inf. Comput. Sci., 34, (1994), pp.1087-1089.

[2] J. A Bondy, U.S.R Murty, Graph Theory with Application, Macmillian press, London, (1976).

[3] J. Baskar Babujee, S. Ramakrishnan, "Topological Indices and New Graph Structures”, Applied Mathematical Sciences, Vol.6, No.108, (2012), pp.5383-5401.

[4] W. Klotz and T. Slander, "Some properties of Unitary Cayley graphs", The Electronic Journal of Combinatorics, 14, (2007), pp.1-12.

[5] Zhihui Cui, Bolian Lui, "On Harary Matrix, Harary Index and Harary Energy", MATCH Commun. Math. Comput. Chem., 68, (2012), pp.815823. 